\title{
THEORY OF A COSMIC SHOCK-WAVE
}

\author{
JOEL SMOLLER* AND BLAKE TEMPLE ${ }^{\dagger}$
}

\begin{abstract}
We discuss mathematical issues related to the authors recent paper "Cosmology with a Shock-Wave" in which we incorporate a shock wave into the standard model of Cosmology. Here we discuss the derivation of the "conservation constraint" which is used to derive the equation for the shock position in that paper.
\end{abstract}

1. Introduction. In Einstein's theory of general relativity, all properties of the gravitational field are determined by the gravitational metric tensor $g$, a Lorentzian metric of signature $(-1,1,1,1)$, defined at each point of a four dimensional manifold $M$ called "spacetime." The equations that describe the time evolution of the metric tensor are given by the Einstein equations, which take the compact form

$$
G=\kappa T
$$

Here $G$ denotes the Einstein curvature tensor, $T$ the stress energy tensor, (the source of the gravitational field) and

$$
\kappa=8 \pi \mathcal{G} / c^{4}
$$

is a universal constant determined by the condition that the theory should incorporate Newton's theory of gravity in the limit of low velocities and weak gravitational fields. Here $c$ denotes the speed of light and $\mathcal{G}$ denotes Newton's gravitational constant. In the case of a perfect fluid, $T$ takes the form

$$
T^{i j}=(\rho+p) u^{i} u^{j}+p g^{i j},
$$

where $u$ denotes the unit 4-velocity, (a tangent vector to a particle path in spacetime), $\rho$ denotes the energy density, (as measured in the inertial frame moving with the fluid), and $p$ denotes the fluid pressure. Here indices always run from 0 to 3 , indices always represent tensor components which can be raised or lowered with the metric, and we assume summation on repeated up-down indices, [33]. The general relativistic version of the compressible Euler equations is given by

$$
\operatorname{Div}(T)=0
$$

where the divergence is taken as the covariant divergence for the metric $g$ so that it agrees with the ordinary divergence in each local inertial coordinate frame, [20]. Equations (1.3) reduce to the relativistic compressible Euler equations in flat Minkowski space, and further reduce to the classical compressible Euler equations in the limit of

*Department of Mathematics, University of Michigan, Ann Arbor, MI 48109, U.S.A. Supported in part by NSF Applied Mathematics Grant Number DMS-92-03972 and the Institute of Theoretical Dynamics, UC-Davis.

$\dagger$ Department of Mathematics, University of California, Davis, Davis CA 95616, U.S.A. Supported in part by NSF Applied Mathematics Grant Number DMS-92-06631 the Institute of Theoretical Dynamics, UC-Davis. 
low velocities. Thus the classical compressible Euler equations of gas dynamics can be viewed as a subsystem of (1.3). Since the covariant derivative depends on the metric components, the conservation equation (1.3) is essentially coupled to the equation for the gravitational field $g$. The Einstein tensor

$$
G_{i j}=R_{i \sigma j}^{\sigma}-\frac{1}{2} R_{\sigma \tau}^{\sigma \tau} g_{i j}
$$

is the simplest tensor constructable from the Riemann curvature tensor $R_{j k l}^{i}$ such that (1.3) follows identically from the Bianchi identities of Riemannian geometry, $\left(R_{j[k l, m]}^{i}=0\right.$, where $[k l, m]$ denotes cyclic sum). Thus (1.3) holds identically on solutions of $G=\kappa T$, a requirement for the consistency of the Einstein equations, [33]. The components of the Riemann curvature tensor in a given coordinate system $x$ are determined from second order derivatives of the metric tensor $g_{i j}(x)$ through the formulas

$$
R_{j k l}^{i}=\Gamma_{j l, k}^{i}-\Gamma_{j k, l}^{i}+\left\{\Gamma_{j l}^{\sigma} \Gamma_{\sigma k}^{i}-\Gamma_{j k}^{\sigma} \Gamma_{\sigma l}^{i}\right\},
$$

where the Christoffel symbols, (connection coefficients), involve first order derivatives of the metric, given by

$$
\Gamma_{j k}^{i}=\frac{1}{2} g^{\sigma i}\left\{-g_{j k, \sigma}+g_{\sigma j, k}+g_{k \sigma, j}\right\} .
$$

The $\Gamma_{j k}^{i}$ are the fundamental objects of classical differential geometry that do not transform like a tensor. Moreover, since tensors transform by first derivatives, second derivatives of tensors are not in general tensors. Thus the Riemman and Einstein curvature tensors are special in that they are obtained from second order derivatives of the metric tensor $g$, but yet remain tensorial.

To summarize, in Einstein's theory of gravity, based on (1.1), the conservation of energy and momentum (1.3) are not imposed, but follow as differential identities from the field equations (1.1). In a specified system of coordinates, (1.1) determines a hyperbolic system of equations that simultaneously describes the time evolution of the gravitational metric, together with the time evolution of the fluid according to (1.3). Since GR is coordinate independent, we can always view the time evolution of (1.1) in local inertial coordinates (coordinates that are "locally flat" in the sense that $g_{i j}=\operatorname{diag}\{-1,1,1,1\}$ and $g_{i j, k}=0$ at a point, [33]) at any point in spacetime, in which case (1.3) reduces to the classical relativistic Euler equations at the point. This tells us that, heuristically, shock-waves must form in the time evolution of (1.1) because one could in principle drive a solution into a shock while in a neighborhood where the equations remained a small perturbation of the classical Euler equations. (This is much easier to say than to demonstrate rigorously, and as far as we know, such a demonstration remains an open problem.)

We now assume that shock-waves are in the time evolution of solutions of the Einstein equations for a perfect fluid, just as they are in the time evolution of solutions of the classical compressible Euler equations. So consider the consequences of (1.1) under the assumption that there are discontinuities in the fluid variables $\rho, u$ and $p$ across some smooth shock surface $\Sigma$. Note that if $T$ is discontinuous across $\Sigma$, then by (1.1), the Einstein curvature tensor $G$ will also have discontinuities across the surface. Since $G$ involves second derivatives of the metric tensor $g$, the only way (1.1) can hold in the classical pointwise a.e. sense at the shock is if the component functions 
$g_{i j}$ are continuously differentiable at the shock, with bounded derivatives on either side, that is, if $g_{i j} \in C^{1,1}$. But it is known that shock-wave solutions of the Einstein equations make sense under the assumption that the metrics match only Lipschitz continuously at the shock surface, that is, $g_{i j} \in C^{0,1}$, [19]. In this case, a condition must be imposed that guarantees that the delta function sources cancel out in $G$ at the shock, so that $G$ on the LHS of (1.1) does not introduce delta function sources of mass and momentum into the RHS of (1.1). (That is, we need a condition that guarantees that the surface defines a true shock-wave, and not a surface layer, [6].) This condition is characterized in the following theorem of [19].

THEOREM 1. Let $\Sigma$ denote a smooth, 3-dimensional shock surface in spacetime with spacelike normal vector $\mathrm{n}$. Assume that the components $g_{i j}$ of the gravitational metric $g$ are smooth on either side of $\Sigma$, (continuous up to the boundary on either side separately), and Lipschitz continuous across $\Sigma$ in some fixed coordinate system. Then the following statements are equivalent:

(i) $[K]=0$ at each point of $\Sigma$.

(ii) The curvature tensors $R_{j k l}^{i}$ and $G_{i j}$, viewed as second order operators on the metric components $g_{i j}$, produce no delta function sources on $\Sigma$.

(iii) For each point $P \in \Sigma$ there exists a $C^{1,1}$ coordinate transformation defined in a neighborhood of $P$, such that, in the new coordinates, (which can be taken to be the Gaussian normal coordinates for the surface), the metric components are $C^{1,1}$ functions of these coordinates.

(iv) For each $P \in \Sigma$, there exists a coordinate frame that is locally Lorentzian at $P$, and can be reached within the class of $C^{1,1}$ coordinate transformations.

Moreover, if any one of these equivalencies hold, then the Rankine-Hugoniot jump conditions, $[G]_{i}^{\sigma} n_{\sigma}=0$, (which express the weak form of conservation of energy and momentum across $\Sigma$ when $G=\kappa T$ ), hold at each point on $\Sigma$.

Here $[K]$ denotes the jump in the second fundamental form (extrinsic curvature) $K$ across $\Sigma$, (this being determined by the metric separately on each side of $\Sigma$ because $g_{i j}$ is only Lipschitz continuous across $\Sigma$ ), and by $C^{1,1}$ we mean that the first derivatives are Lipschitz continuous.

For spherically symmetric solutions, it follows that the above equivalencies reduce to the single condition

$$
[G]_{\sigma \tau} n^{\sigma} n^{\tau}=0
$$

so long as there is a smooth matching of the spheres of symmetry at the shock, [19].

We now apply (1.6) to the problem of matching the standard FriedmannRobertson-Walker (FRW) metric of Cosmology to a static Tolman-OppenheimerVolkoff (TOV) metric across a shock-wave. The FRW line element is given by

$$
d s^{2}=-d t^{2}+R(t)^{2}\left(\frac{d r^{2}}{1-k r^{2}}+r^{2} d \Omega^{2}\right),
$$

and the TOV line element is given by

$$
d s^{2}=-B(r) d t^{2}+\left(1-\frac{2 G M(r)}{r}\right)^{-1} d r^{2}+r^{2} d \Omega^{2}
$$


Here $d \Omega^{2}=d \theta^{2}+\sin ^{2}(\theta) d \phi^{2}$ denotes the standard line element on the unit 2-sphere, $\mathcal{G}$ denotes Newton's gravitational constant, $M(r)$ denotes the total mass inside radius $r$, and $B(r)$ is a function that tends smoothly to $1-2 \mathcal{G} M(r) / r$ as the density vanishes. The only unknown in the FRW metric is the cosmological scale factor $R(t)$ from which the Hubble "constant" $H$ is determined,

$$
H=\frac{\dot{R}(t)}{R(t)}
$$

The FRW metric describes the time evolution of a three dimensional space of constant scalar curvature, (the $t=$ constant surfaces), and the sign of the curvature is given by $\operatorname{sign}(k)$, a constant that can be rescaled to one of the values $\{-1,0,1)$ via a rescaling of the radial coordinate $r$. Of course, the line element determines the metric components $g_{i j}$ through the identity

$$
d s^{2}=g_{i j} d x^{i} d x^{j}
$$

We now outline the procedure for matching an FRW metric (1.7) to a TOV metric (1.8) across a shock interface. This requires defining a coordinate transformation $(t, r) \rightarrow(\bar{t}, \bar{r})$ such that, under this coordinate identification, (take the TOV coordinates as barred), the metrics match Lipschitz continuously, and the conservation condition (1.6) holds across the interface, [19]. The matching proceedure goes as follows: First, the matching of the spheres of symmetry implies the identity

$$
\bar{r}=R(t) r
$$

Using this, one can go to $(t, \bar{r})$ coordinates and match the $g_{\bar{r} \bar{r}}$ coordinates. After simplification, this implies the identity

$$
M(\bar{r})=\frac{4 \pi}{3} \rho(t) \bar{r}^{3}
$$

This implicitly defines the shock position $\bar{r}=\bar{r}(t)$, that is, the TOV position $\bar{r}$ is given as a function of FRW time $t$. Having (1.11) enables one to match the $g_{t t}$ components at the implicitly defined surface, and this leads to the fact that the function $\bar{t}(t, r)$ solves a linear PDE with initial conditions chosen so that Lipschitz matching of the metrics is achieved at the shock surface. It follows that the shock surface is non-characteristic for this PDE as long as the shock lies outside the Schwarzschild radius. From this one concludes that the coordinate identification is defined in a neighborhood of the shock surface, and the Lipschitz matching of an arbitrary FRW and TOV metric is achieved. Only the $\bar{t}(t, r)$ mapping is not given explicitly, but it turns out that only its existence is important for the further development. It remains, however, to impose the additional conservation constraint (1.6). On solutions of $G=\kappa T$, the condition (1.6) is equivalent to the condition

$$
\left[T^{i j}\right] n_{i} n_{j}=0 .
$$

A calculation using the identities (1.2), (1.10) and (1.11) leads to the following equivalent formulation of the conservation constraint: 


$$
0=(1-\theta)(\rho+\bar{p})(p+\bar{\rho})^{2}+\left(1-\frac{1}{\theta}\right)(\bar{\rho}+\bar{p})(\rho+p)^{2}+(p-\bar{p})(\rho-\bar{\rho})^{2}
$$

where

$$
\theta=\frac{A}{1-k r^{2}}
$$

Note that this is a homogeneous polynomial in the $\rho^{\prime} s$ and $p^{\prime} s$.

Now one can use the identity (1.13) alone to derive the exact shock-wave solutions first constructed in [20]. The idea is that there exists exact FRW solutions for equation of state

$$
p=\sigma \rho
$$

and other exact TOV solutions with equation of state

$$
\bar{p}=\bar{\sigma} \bar{\rho},
$$

where $\sigma$ and $\bar{\sigma}$ are assumed to be constant, (modeling the simple case of a constant sound speed). In this special case, the exact TOV solutions have $A=$ constant, and the FRW solutions have $k=0$, so it follows that because $\theta=$ constant and (1.12) is homogeneous cubic, when the $p^{\prime} s$ are linearly related to the $\rho^{\prime} s$ as in (1.14) and $(1.15),(1.12)$ reduces to a constraint that gives a cubic relation between $\sigma$ and $\bar{\sigma}$; that is, a relation between the inner and the outer sound speeds. In fact, the constraint can be reduced to the explicit form

$$
\bar{\sigma}=H(\sigma) \equiv \frac{1}{2} \sqrt{9 \sigma^{2}+54 \sigma+49}-\frac{3}{2} \sigma-\frac{7}{2} .
$$

(See [20] for details.) The point is that no more than the condition (1.12) is required to verify conservation in the construction of these examples when the sound speed is constant, and the conservation constraint imposes a relation between the inner and outer sound speeds at the shock. The discovery of the explicit formula for the function $H(\sigma)$ above required (MAPLE) finding that the conservation constraint (1.12) factors, and the factoring of this cubic polynomial is the key to using this method to incorporate a shock-wave into the standard model of cosmology.

So consider now the problem of matching a TOV metric on the outside of an expanding FRW metric when the pressure and energy density agree with those of the standard model of cosmology after the time $t_{*}$ at which the thermal uncoupling of radiation and matter occured, where, $t_{*} \approx 300,000$ years after the Big Bang, and at a temperature of about $T_{*} \approx 4000^{\circ} \mathrm{K}$. The idea is that radiation is in thermal equilibrium with matter up until the time when the universe became transparent, and after this time, the radiation evolves with a blackbody spectrum associated with a temperature $T$ that is inversely proportional to the FRW scale factor $R$,

$$
T=\frac{R_{*}}{T_{*}} \frac{1}{R} .
$$


After time $t_{*}$, the pressure of matter can be neglected, and so in the standard model, the energy density of the universe is the sum of the contribution from the matter and radiation separately, and the the pressure is due to the pressure of radiation alone, as determined by the Stefan-Boltzmann law. It follows from these assumptions, together with the FRW equations,

$$
\dot{R}^{2}=\frac{8 \pi \mathcal{G}}{3 c^{4}} \rho R^{2}-k
$$

and

$$
\frac{d}{d R}\left(\rho R^{3}\right)=-3 p R^{2}
$$

(these are the Einstein equations when one takes (1.7) as the ansatz for the metric, "dot" denotes $d / d(c t))$, that the total energy density and pressure due to radiation plus matter in the universe after time $t=t_{*}$ is given in terms of the FRW scale factor by the formulas, [27],

$$
Q=\frac{3 \alpha}{R^{4}}+\frac{\beta}{R^{3}}, \quad P=\frac{\alpha}{R^{4}}
$$

where $Q$ and $P$ are given in terms of the physical energy density and pressure through the formulas

$$
Q=\frac{8 \pi \mathcal{G}}{3 c^{4}} \rho, \quad P=\frac{8 \pi \mathcal{G}}{3 c^{4}} p
$$

and

$$
\rho=\rho_{\text {radiation }}+\rho_{\text {matter }}, \quad p=p_{\text {radiation }} .
$$

The constants $\alpha$ and $\beta$ are easily determined from the present value of the radiation temperature,

$$
T_{0} \approx 2.7^{\circ} \mathrm{K}
$$

and the Hubble constant,

$$
H_{0}=h_{0} \frac{100 k m}{s \cdot m p c}
$$

where $h_{0}$ is believed to be in the range $.5 \leq h_{0} \leq .85$. Note that once one has (1.19), one can substitute these values into (1.17) to obtain an equation for the FRW scale factor $R$ as a function of time $t$ for $t \geq t_{*}$. In particular, the FRW metric for standard cosmology is given in closed form when $R$ is taken as the independent variable.

Consider now the problem of matching this FRW metric to a TOV metric across a shock surface, such that the conservation constraint (1.12) holds across the surface. It is difficult to apply (1.12) directly. What makes this possible is that the homogeneous cubic polynomial in (1.12) factors, thus allowing one to solve for two different possible 
TOV pressures, one of which can be ruled out on physical grounds. Indeed, the factoring of (1.12) leads to two factors, each of which can be solved for the FRW pressure, leading to the following formulas for two possible pressures, $p_{+}$and $p_{+}$, given as a function of $\rho, \bar{\rho}$ and $\bar{p}$, these representing a reformulation of the conservation constraint, (unbarred refers to FRW values and barred refers to TOV values, at the shock, [19]):

$$
p_{+}=\frac{\frac{1}{2}\left\{-(\bar{\rho}+\rho)^{2}+2(\theta-1) \bar{\rho} \bar{p}+2\left(\theta+\frac{1}{\theta}\right) \rho \bar{\rho}+2\left(\frac{1}{\theta}-1\right) \rho \bar{p} \pm S Q\right\}}{(1-\theta) \rho+\left(2-\theta-\frac{1}{\theta}\right) \bar{p}+\left(1-\frac{1}{\theta}\right) \bar{\rho}}
$$

where

$$
S Q=\left(6 \bar{\rho}^{2} \rho^{2}-4 \rho^{3} \bar{\rho}-4 \overline{\rho^{3}} \rho+\rho^{4}+\bar{\rho}^{4}\right)^{1 / 2}=(\rho-\bar{\rho})^{2} .
$$

Simplification using identities at the shock that come from the Lipschitz matching of the metrics, leads to the following simpler formulas for $p_{-}$and $p_{+}$:

$$
\begin{aligned}
& p_{+}=\frac{\Theta \bar{\rho}-\rho}{1-\Theta}, \\
& p_{-}=\frac{\theta \bar{\rho}-\rho}{1-\theta},
\end{aligned}
$$

where

$$
\Theta \equiv \gamma \theta
$$

and

$$
\gamma \equiv \frac{\rho+\bar{p}}{\bar{\rho}+\bar{p}}
$$

An easy calculation gives a symmetrical formulation of (1.24) in terms of the TOV pressure $\bar{p}$,

$$
\bar{p}=\frac{\theta \bar{\gamma} \rho-\bar{\rho}}{1-\theta \bar{\gamma}}
$$

where

$$
\bar{\gamma}=\frac{\bar{\rho}+p}{\rho+p} .
$$

Now if we interpret the FRW-TOV shock-wave as the leading edge of an explosion in which the FRW solution is on the inside, expanding outward into the static TOV solution, then as an entropy condition we can take $\bar{\rho} / \rho<1$; that is, the density should be greater behind the shock. In [19] we show that this, (together with the condition that pressure should increase with density), leads to the conclusion that we must take the pressure to be $p=p_{+}$, in which case (1.24), or its equivalent form (1.28), must be imposed for conservation. 
To get the final equations for the shock position and TOV metric that matches the standard FRW metric of cosmology across a shock wave, we differentiate the shock position equation

$$
M(\bar{r})=\frac{4 \pi}{3} \rho(t) \bar{r}(t)^{3},
$$

with respect to $t$ and use the pressure constraint (1.24), together with identities arising from the matching of the metrics, to get the following equivalent formulation of (1.24) (details omitted),

$$
\dot{r}=\frac{1}{R}\left(\frac{p-\bar{p}}{\rho+\bar{p}}\right) \frac{1-k r^{2}}{\dot{R} r} .
$$

This can be coupled with the Oppenheimer-Volkoff equation, the constraint on the TOV pressure that comes from the Einstein equations, namely,

$$
-\bar{r}^{2} \frac{d \bar{p}}{d \bar{r}}=\mathcal{G} M \bar{\rho}\left\{1+\frac{\bar{p}}{\bar{\rho}}\right\}\left\{1+\frac{4 \pi \bar{r}^{3} \bar{p}}{M}\right\}\left\{1-\frac{2 \mathcal{G} M}{\bar{r}}\right\}^{-1} .
$$

Rewriting (1.30) and (1.31) so that the FRW scale factor $R$ is taken as the independent variable, and simplifying, yields the following system of two equations that close when (1.19) are taken as the constitutive assumptions:

$$
\begin{aligned}
& \frac{d r}{d R}=\frac{R}{\left(3 \alpha+\beta R-k R^{2}\right)}\left(\frac{\alpha-\bar{P} R^{4}}{3 \alpha+\beta R+\bar{P} R^{4}}\right) \frac{\left(1-k r^{2}\right)}{r} \\
& \frac{d \bar{P}}{d R}=-\frac{1}{2 R^{5}} \frac{\left(3 \alpha+\beta R+3 \bar{P} R^{4}\right)\left(\alpha-\bar{P} R^{4}\right)}{3 \alpha+\beta R-k R^{2}}
\end{aligned}
$$

Observe, again, that a nice feature of the formulation (1.32), (1.33) is that, in this formulation, the second equation (1.33) for the TOV pressure $\bar{P}$ uncouples from the first equation (1.32) for the shock position $r$. One can finally obtain a formula for $\bar{Q}$ by using the second TOV equation

$$
M^{\prime}(\bar{r})=4 \pi \bar{\rho} \bar{r}^{2}
$$

A calculation leads to the formula

$$
\bar{Q}=\frac{1}{3 \bar{r}^{2}} \frac{d}{d \bar{r}}\left(Q \bar{r}^{3}\right)=Q+\frac{R r}{3} \frac{d}{d \bar{r}} Q
$$

Note that if $Q$ decreases as the shock moves outward, (that is, the $\bar{r}$ position of the shock increases), then the second term in (1.34) is negative, and so $\bar{Q}<Q$, (the density behind the shock is greater than the density in front of the shock), as is the case for classical shock-waves in fluids, [17]. Note, however that the physically necessary condition $\bar{Q}>0$, or the physically reasonable condition $\bar{Q}>\bar{P}$, is not guaranteed. This condition puts an outer bound on the possible shock position, and is related to the fact that if the shock-wave is much beyond the Hubble distance from the center of the explosion, then in fact the expanding universe is inside its Schwarzschild radius, where the TOV system breaks down since there are no spherical static solutions inside an event horizon. For further details, we refer the reader to the authors' paper [27], where the simplest case $k=0$ is worked out in detail. 


\section{REFERENCES}

[1] S.K. Blau And A.H. Guth, Inflationary cosmology. In: Three Hundred Years of Gravitation, ed. by S.W. Hawking and W. Israel, Cambridge University Press, 1987, pp. 524-603.

[2] R. Courant and K. Friedrichs, Supersonic Flow and Shock-Waves, Wiley-Interscience, 1948. 1972.

[3] A. Einstein, Der feldgleichungen der gravitation, Preuss. Akad. Wiss., Berlin, Sitzber. 1915b, pp. 844-847. 314 (1970), pp. 529-548.

[4] S.W. HaWking and G.F.R. Ellis, The Large Scale Structure of Spacetime, Cambridge University Press, 1973.

[5] S.W. HAWKIng AND R. PENRose, The singularities of gravitational collapse and cosmology, Proc. Roy. Soc. Lond. A, 314 (1970), pp. 529-548.

[6] W. IsRael, Singular hypersurfaces and thin shells in general relativity, IL Nuovo Cimento, Vol. XLIV B, No. 1, 1966, pp. 1-14.

[7] W. IsRaEL, Dark stars: the evolution of an idea, in: 300 years of gravitation, edited by S. W. Hawking and W. Israel, Cambridge University Press, 1987, pp. 199-276.

[8] P.D. LAX, Hyperbolic systems of conservation laws, II, Comm. Pure Appl. Math., 10 (1957), pp. $537-566$.

[9] M.S. Longair, Our Evolving Universe, Cambridge University Press, (1996).

[10] G.C. MCVitTie, Gravitational collapse to a small volume, Astro. Phys. Jour., 140 (1964), pp. 401-416.

[11] C. Misner AND D. Sharp, Relativistic equations for adiabatic, spherically symmetric gravitational collapse, Phys. Rev., 26 (1964), pp. 571-576.

[12] C. Misner, K. Thorne, And J. Wheeler, Gravitation, Freeman, 1973.

[13] J.R. OPPENheImer AND J.R. SNYder, On continued gravitational contraction, Phys. Rev., 56 (1939), pp. 455-459.

[14] J.R. OpPenheimer AND G.M. Volkoff, On massive neutron cores, Phys. Rev., 55 (1939), pp. 374-381.

[15] P.J.E. Peebles, Principles of Physical Cosmology, Princeton University Press, 1993.

[16] R. Schoen And S.T. YAU, Proof of the positive mass theorem II, Commun. Math. Phys, 79 (1981), pp. 231-260.

[17] J. SMOLLER, Shock-Waves and Reaction-Diffusion Equations, Springer Verlag, 1983.

[18] J. Smoller And B. Temple, Global solutions of the relativistic Euler equations, Comm. Math. Phys., 157 (1993), pp. 67-99.

[19] J. Smoller AND B. TEmPle, Shock-wave solutions of the Einstein equations: the OppenheimerSnyder model of gravitational collapse extended to the case of non-zero pressure, Arch. Rat. Mech. Anal., 128 (1994), pp. 249-297.

[20] J. Smoller AND B. Temple, Astrophysical shock-wave solutions of the Einstein equations, Phys. Rev. D, 51, No. 6 (1995).

[21] J. Smoller And B. Temple, General relativistic shock-waves that extend the OppenheimerSnyder model, Arch. Rat. Mech. Anal., 138 (1997), pp. 239-277.

[22] J. Smoller AND B. Temple, Shock-waves near the Schwarzschild radius and the stability limit for stars, Phys. Rev. D, 55 (1997), pp. 7518-7528.

[23] J. Smoller AND B. Temple, Shock-wave solutions in closed form and the Oppenheimer-Snyder limit in general relativity, with J. Smoller, SIAM J. Appl. Math., Vol. 58, No. 1, pp. 15-33, February, 1998.

[24] J. Smoller And B. Temple, On the Oppenheimer-Volkov equations in general relativity, with J. Smoller, Arch. Rat. Mech. Anal., 142 (1998), pp. 177-191.

[25] J. Smoller AND B. Temple, Solutions of the Oppenheimer-Volkoff equations inside $9 / 8$ 'ths of the Schwarzschild radius, with J. Smoller, Commun. Math. Phys., 184 (1997), pp. 597-617.

[26] J. SMOller AND B. Temple, Shock-waves near the Schwarzschild radius and the stability limit for stars, with J. Smoller, Physical Review D, Vol. 55, No. 12 (1997), pp. 7518-7528.

[27] J. Smoller And B. Temple, Cosmology with a shock-wave, Comm. Math. Phys., 210 (2000), pp. 275-308.

[28] J. Smoller AND B. Temple, Shock-wave solutions of the Einstein equations: A general theory with examples, Third Annual Summer School, Training and mobility of Researchers Program, European Union, Kochel, Germany, 1999, ed. by H. Freisthuler, and A. Szeoessy, Birkhauser, (2001), pp. 105-258.

[29] J. Groah AND B. Temple, A shock-wave formulation of the Einstein equations, Methods in Appl. of Anal., 7 (2000), pp. 793-812.

[30] R. Tolman, Static Solutions of Einstein's Field Equations for Spheres of Fluid, Physical Review, 55 (1939), pp. 364-374. 
[31] R. Tolman, Relativity, Thermodynamics and Cosmology, Oxford University Press, 1934.

[32] R.M. WALD, General Relativity, University of Chicago Press, 1984.

[33] S. Weinberg, Gravitation and Cosmology: Principles and Applications of the General Theory of Relativity, John Wiley \& Sons, New York, 1972. 